\title{
Genetic Diversity in Tomato Genotypes (Solanum lycopersicum) Based on Salinity Responsive Candidate Gene Using Simple Sequence Repeats
}

\author{
Umar Ja'afar ${ }^{1, *}$, A.A. Aliero ${ }^{2}$, K. Shehu ${ }^{1}$, L. Abubakar ${ }^{3}$ \\ ${ }^{1}$ Department of Biology, Federal University, Birnin Kebbi, Nigeria \\ ${ }^{2}$ Department of Biological Sciences, Usmanu Danfodiyo University, Sokoto, Nigeria \\ ${ }^{3}$ Department of Crop Science, Usmanu Danfodiyo University, Sokoto, Nigeria \\ realumar2001@gmail.com
}

Keywords: Tomato, candidate gene, salt responsive gene, DNA, dendrogram.

\begin{abstract}
Salinity inhibition of plant growth is the result of osmotic and ionic effect and different plant species have developed different mechanisms to cope with those effects. With the discovery of molecular markers and marker assisted selection technology, it is possible to develop markers that identify salt tolerance. The genetic diversity of tomato genotypes were analyzed using SSRs polymorphic markers and Unweighted Pair Group Method with Arithmetic Mean. Leaves of the twenty tomato genotypes (landraces/accessions in Nigeria) were used to isolate their DNA using Bioland Plant Genomic DNA protocols. Primers were designed from 15 different salt responsive candidate genes, using Vector NTI and the sequence of the genes were obtained from ncbi genomic web site. All 15 primers sets generated shows clear distinct polymorphic profiles as evident from the $6 \%$ agarose gel profile. Dendrogram generated shows three groups, none of the panel intermixed in a subgroup. The genetic distance information reported in this study might be used by breeders when planning future crosses among tomato genotypes. From the result obtained UC82B recorded the highest vegetative and yield parameters, therefore, adoption of this genotype could be help to increase the tomato production in Sokoto agro-climatic area.
\end{abstract}

\section{Introduction}

Tomato (Solanum lycopersicum L.), belong to the Solanaceae family which is one of the most important vegetables being widely grown in both fields and under protected cultivation. Most tomato cultivars are sensitive to moderate levels of salinity [1]. Indeed, all plant development stages, includingseed germination, vegetative growth and reproduction, show salinity sensitivity, that leads to poor harvests and reduced economic yield [2]. Tomato is considered as a vegetable model and has thus been subjected to molecular investigation resulting in abundant genomic information (http://solgenomics.net/). In addition to its worldwide agricultural and economic importance as a crop, tomato is a pre-eminent model system for genetic studies in plants.

The use of molecular markers in breeding by means of marker assisted selection (MAS) could improve performance under extreme environments [3]. Tomato (Solanum lycopersicum L.), a major horticultural crop consumed all over the world, suffers heavy losses due to salinity. USP (universal stress protein) family proteins, first identified in prokaryotes, appear to play an active role in abiotic stress response, but their function remains largely unknown in plants [4]. A USP gene (SpUSP), cloned from wild tomato (S. pennellii) and functionally characterized in cultivated tomato exhibited increased expression under dehydration stress, salinity, oxidative stress and phyto-hormone ABA treatment. With the discovery of molecular markers and marker assisted selection technology, research has entered in to a new era and has made it possible to develop new and more informative PCR-based markers, including simple sequence repeats (SSRs), and to further facilitate the use of markers in tomato breeding. Genomic microsatellite markers are an elite group of markers, but there is possible uncertainty of linkage with the important genes. In contrast, there are better possibilities of linkage detection with important genes if SSRs are developed from candidate genes [5]. 
Deoxyribonucleic acid (DNA) polymorphisms provide a powerful tool for quantifying the existing levels of genetic variation in plant germplasm [6]. Molecular markers can provide an effective tool for efficient selection of desired agronomic traits because they are based on the plant genotypes and thus, are independent of environmental variation. It is suggested that the variation or polymorphism of SSRs are as a result of polymerase slippage during DNA replication or unequal crossing over [7]. SSRs are not only very common, also are hyper variable for numbers of repetitive DNA motifs in the genomes of eukaryotes [8-9]. Development of SSR markers based on QTL or candidate genes related to an important agronomic trait is useful in marker-assisted breeding programs for the concerned trait. In line with this, SSR markers were combined with morphological traits to assess the genetic diversity of cultivated and wild tomatoes [10]. The use of molecular markers can facilitate tomato breeding by means of marker assisted selection (MAS) to improve important agronomical traits such as yield, fruit quality, and disease resistance.

\section{Theory}

There are various detrimental effects of salinity in crop plants, which impose severe decrease in growth and yield of plants. About $90 \%$ of the farmers complained of salinity, water logging, soil erosion, degradation, sedimentation, build up of pests and diseases as a result of irrigation related problems [11]. Over 45\% (750,000 metric tons) of tomatoes produced in Nigeria is estimated as annual loss due to poor irrigation, abiotic stress, price instability resulting from seasonal fluctuation in production [12]. One of the pioneer reports on study of water quality in northwestern Nigeria was made in 1962 which reported that waters from Rima, Sokoto and Zamfara rivers had low to moderate salt content although some of the waters contained principally sodium and bicarbonates [13]. Majority of the farmers in Sokoto valley are peasant farmers and engage in both rain fed and traditional irrigation farming in the dry season. They accounted for the bulk of vegetables and spices produced in the area which include among others onion, garlic and tomatoes.

\section{Materials and Methods}

\section{Plant Materials}

Selected tomato landraces genotypes were obtained from local markets around Sokoto and Zamfara metropolis. These were identified in Herbarium of Ahmadu Bello University, Zaria. While accessions genotypes seeds were obtained from Zamfara State Agricultural Development Project, Gusau (ZADP) and Institute for Agricultural Research (IAR) Ahmadu Bello University Zaria. The seeds of 20 genotypes of tomato were grouped into landraces and accessions, the collection location, type and common name of the cultivar are summarized on Table 1. 
Table 1. List of Tomato Genotypes used.

\begin{tabular}{|c|c|c|c|c|c|c|c|c|}
\hline S/No. & Genotype & Type & Source & $\begin{array}{l}\text { Growth } \\
\text { habit }\end{array}$ & $\begin{array}{l}\text { Fruit } \\
\text { shape }\end{array}$ & Fruit size & Colour & Leaf type \\
\hline 1 & Dangainakawa & Landrace & $\begin{array}{l}\text { Mafara } \\
\text { Market }\end{array}$ & Determinate & Pyriform & Very small & Red & Dwarf \\
\hline 2 & Bahaushe & Landrace & $\begin{array}{l}\text { Kasuwar } \\
\text { tashar } \\
\text { Illela }\end{array}$ & Indeterminate & $\begin{array}{l}\text { Slightly } \\
\text { flattened }\end{array}$ & Intermediate & Red & $\begin{array}{l}\text { Potato leaf } \\
\text { type }\end{array}$ \\
\hline 3 & Dandino & Landrace & $\begin{array}{l}\text { Mafara } \\
\text { Market }\end{array}$ & Indeterminate & $\begin{array}{l}\text { High } \\
\text { round }\end{array}$ & Small & Red & $\begin{array}{l}\text { Potato leaf } \\
\text { type }\end{array}$ \\
\hline 4 & Dan Eka & Landrace & $\begin{array}{l}\text { Mafara } \\
\text { Market }\end{array}$ & Indeterminate & Pyriform & Intermediate & Red & $\begin{array}{l}\text { Potato leaf } \\
\text { type }\end{array}$ \\
\hline 5 & Dan Gombe & Landrace & $\begin{array}{l}\text { Kasuwar } \\
\text { tashar } \\
\text { Illela }\end{array}$ & Indeterminate & $\begin{array}{l}\text { Slightly } \\
\text { flattened }\end{array}$ & Small & Red & $\begin{array}{l}\text { Potato leaf } \\
\text { type }\end{array}$ \\
\hline 6 & Dan mazari & Landrace & $\begin{array}{l}\text { Shinkafi } \\
\text { Market }\end{array}$ & Indeterminate & Oblong & Small & Red & $\begin{array}{l}\text { Potato leaf } \\
\text { type }\end{array}$ \\
\hline 7 & $\begin{array}{l}\text { Dan dubu } \\
\text { kamiya }\end{array}$ & Landrace & $\begin{array}{l}\text { Jangebe } \\
\text { Market }\end{array}$ & Determinate & Rounded & Small & $\begin{array}{l}\text { Yellow- } \\
\text { orange }\end{array}$ & $\begin{array}{l}\text { Potato leaf } \\
\text { type }\end{array}$ \\
\hline 8 & $\begin{array}{l}\text { Dan } \\
\text { Kwandawa }\end{array}$ & Landrace & $\begin{array}{l}\text { Achida } \\
\text { Market }\end{array}$ & Determinate & $\begin{array}{l}\text { Slightly } \\
\text { flattened }\end{array}$ & Intermediate & Red & $\begin{array}{l}\text { Potato leaf } \\
\text { type }\end{array}$ \\
\hline 9 & Ganwon Falke & Landrace & $\begin{array}{l}\text { Achida } \\
\text { Market }\end{array}$ & Determinate & Flattened & Large & Red & $\begin{array}{l}\text { Potato leaf } \\
\text { type }\end{array}$ \\
\hline 10 & $\begin{array}{l}\text { Dan } \\
\text { Dogarawa }\end{array}$ & Landrace & $\begin{array}{l}\text { Shinkafi } \\
\text { Market }\end{array}$ & Indeterminate & $\begin{array}{l}\text { Slightly } \\
\text { flattened }\end{array}$ & Intermediate & red & $\begin{array}{l}\text { Potato leaf } \\
\text { type }\end{array}$ \\
\hline 11 & Roma & Commercial & ZADP & Determinate & Ellipsoid & Intermediate & Orange & $\begin{array}{l}\text { Potato leaf } \\
\text { type }\end{array}$ \\
\hline 12 & UTC & Commercial & ZADP & Indeterminate & Globe & Intermediate & Red & $\begin{array}{l}\text { Potato leaf } \\
\text { type }\end{array}$ \\
\hline 13 & Rio grande & Commercial & ZADP & Determinate & $\begin{array}{l}\text { High } \\
\text { rounded }\end{array}$ & Intermediate & Red & Pervianum \\
\hline 14 & Giofranco F & Commercial & ZADP & Determinate & $\begin{array}{l}\text { Slightly } \\
\text { flattened }\end{array}$ & Intermediate & Red & $\begin{array}{l}\text { Potato leaf } \\
\text { type }\end{array}$ \\
\hline 15 & UC82B & Commercial & ZADP & Determinate & Globe & Intermediate & Red & $\begin{array}{l}\text { Potato leaf } \\
\text { type }\end{array}$ \\
\hline 16 & Indian tomato & Commercial & IAR & Indeterminate & Oblong & Small & Red & $\begin{array}{l}\text { Potato leaf } \\
\text { type }\end{array}$ \\
\hline 17 & Tomato peto & Commercial & IAR & Determinate & Ellipsoid & Intermediate & Brick red & Bipinnate \\
\hline 18 & Tropimech & Commercial & IAR & Indeterminate & Rounded & Intermediate & Red & Hirsutum \\
\hline 19 & Cherry & Commercial & IAR & Indeterminate & Rounded & Small & $\begin{array}{l}\text { Yellowish- } \\
\text { red }\end{array}$ & $\begin{array}{l}\text { Potato leaf } \\
\text { type }\end{array}$ \\
\hline 20 & Heirloom & Commercial & IAR & Indeterminate & Flattened & Intermediate & Purple & $\begin{array}{l}\text { Potato leaf } \\
\text { type }\end{array}$ \\
\hline
\end{tabular}

\section{Phenotypic Evaluation}

Plant height, root length, leaf area and dry matter accumulations were computed according to International plant genetic resource institute manuals [14].

\section{Isolation of Genomic DNA}

Fresh green leaves were collected from twenty selected tomato plant samples and weighed (100 mg), in $2.0 \mathrm{~mL}$ micro centrifuge tube and immediately $600 \mu 1 \mathrm{Buffer}$ PL. 1 was added. The DNA was isolated following a protocol of Bioland Plant Genomic DNA.

\section{Retrieval of Salt Tolerant Gene Sequences, Simple Sequence Repeats Detection and Primer Design}

Nucleotide sequences conferring salt tolerance in tomato were downloaded from National Center for Biotechnology Information (NCBI). The downloaded nucleotide sequences were used to mine simple sequence repeats. The gene sequences were used to mine SSRs in SSR identification tool. Respective references of those candidate genes which have been found to contain 
microsatellite repeats were used. Primers was designed manually with the following parameters: primer length $18-30 \mathrm{bp}$, melting temperature $50-60^{\circ} \mathrm{C}$, GC percentage $40-60$ and product size160-500bp using Vector NTI software [15].

\section{PCR Amplification and 3\% Agarose Gel Electrophoresis}

PCR amplification was performed on 20 genotypes with 15 pairs of SSR primers in a total volume of $25 \mu \mathrm{l}$ using a C1000 Thermal Cycler (Bio Rad, USA). Each $25 \mu$ l volume of reaction mixture contained $50 \mathrm{ng}$ of genomic DNA as template, $1 \mathrm{X}$ Taq polymerase buffer, $2 \mathrm{mM} \mathrm{MgCl}$, $0.2 \mathrm{mM}$ dNTPs mix, $0.4 \mathrm{pM}$ each of the forward and reverse primer, $1 \mathrm{U}$ of Taq polymerase. The optimized condition was initial 5 minutes incubation at $97^{\circ} \mathrm{C}$ for complete denaturation, followed by 38 cycles consisting of $94^{\circ} \mathrm{C}$ for $1 \mathrm{~min}, 55^{\circ} \mathrm{C}-60^{\circ} \mathrm{C}$ (vary with the primer pair) for $1 \mathrm{~min}, 72^{\circ} \mathrm{C}$ for $2 \mathrm{~min}$ and finally $72^{\circ} \mathrm{C}$ for $10 \mathrm{~min}$. The experiments were repeated twice. Resolving of all PCR products were performed in a vertical non denaturing 3\% Agrose gel electrophoresis system at constant $90 \mathrm{~V}$ with $1 \mathrm{X}$ TAE (Tris acetate EDTA) buffer (pH-8.0). The gel was stained with ethidium bromide solution and visualized in gel documentation system (Protein Simple, USA) adopting the methods of [16].

\section{Allele Scoring}

Molecular weights of the amplified bands were determined by the number of base pair were multiplied by the average molecular mass of one base pair $(660 \mathrm{~g} / \mathrm{mol})$ to get the approximate mass of the whole double-stranded DNA molecule. Molecular weights of the amplified bands were determined based on the relative migration of standard $100 \mathrm{bp}$ DNA ladder (Thermo Scientific, USA) in the gel. Presence and absence of a particular allele was denoted as 1 or 0 respectively. Allele exclusively found in one genotype, it was designated as unique allele, in less than $5 \%$ of genotypes were designated as rare [15].

\section{Results}

\section{Phenotypic Responses of Tomato to Salt Stress}

Salinity significantly reduced plant vegetative parameters (number of leaves, plant height, root length, leaves area and Dry Matter Accumulation) in concentration dependent manner in all the twenty genotype. The highest number of leaves were recorded in control of Riogrande and Daneka with 88.67 leaves per plant each and the lowest leaves count were recorded in Dankwandawa and Dan gainakawa treated with $60 \mathrm{mgL}-1$ of salt with 16.67 and 17.00 leaves per plant (Table 2). However, Plant height significantly $(\mathrm{P}<0.05)$ affected by salt concentration in all the genotype used. Daneka recorded the highest plant height of 61.00 at control and Dandubukamiya recorded the highest plant height in plant treated with $60 \mathrm{mgL}-1$ of salt with $38.67 \mathrm{~cm}$ (Table 2 ).

Root length significantly affected by salinity in concentration dependant manner. However, the lowest length of root at control of $3.03 \mathrm{~cm}$ was recorded in Dankwandawa followed by Ganwon falke with $3.70 \mathrm{~cm}$. The highest root length in plants treated with $30 \mathrm{mgL}-1$ of Salinity was recorded in Daneka with $10.17 \mathrm{~cm}$ and the lowest root length in plant treated with $30 \mathrm{mgL}-1$ of Salinity was observed in UTC and Dandogarawa with $2.27 \mathrm{~cm}$ each (Table 2). Leaf area also significantly $(\mathrm{P}<0.05)$ affected by salinity. The largest leave area of $27.30 \mathrm{sq} / \mathrm{m}$ was recorded in Tropimech followed by Roma with 24.67sq/m (Table 2). Dry Matter Accumulation significantly affected by salinity episode in 
Table 2. Effects of Salt Concentrations on Phenotypic Parameters in Tomato genotypes.

\begin{tabular}{|c|c|c|c|c|c|c|}
\hline Genotypes & $\begin{array}{l}\text { Treatment } \\
\left(\mathrm{mgL}^{-1}\right)\end{array}$ & $\begin{array}{l}\text { Number } \begin{array}{r}\text { of } \\
\text { leaves } \\
\text { plant) }\end{array} \\
\end{array}$ & $\begin{array}{l}\text { Plant } \\
\text { Height } \\
\text { (cm/plant) }\end{array}$ & $\begin{array}{l}\text { Root length } \\
\text { (cm/plant) }\end{array}$ & $\begin{array}{l}\text { Leaf area } \\
(\mathrm{sq} / \mathrm{m})\end{array}$ & $\begin{array}{l}\text { Dry matter } \\
\text { accumulation } \\
\text { (g/plant) }\end{array}$ \\
\hline \multirow{4}{*}{ Dan gainakawa } & 0 & $79.00^{\mathrm{a}} \pm 21.78$ & $45.67^{\mathrm{a}} \pm 2.50$ & $13.67^{\mathrm{a}} \pm 5.51$ & $6.30^{\mathrm{a}} \pm 0.57$ & $4.60^{\mathrm{a}} \pm 0.66$ \\
\hline & 30 & $45.00^{\mathrm{b}} \pm 12.10$ & $40.67^{b} \pm 1.16$ & $7.67^{\mathrm{b}} \pm 0.58$ & $5.30^{\mathrm{b}} \pm 0.57$ & $1.97^{\mathrm{b}} \pm 0.58$ \\
\hline & 60 & $17.00^{c} \pm 15.13$ & $9.18^{\mathrm{c}} \pm 10.00$ & $4.50^{c} \pm 3.90$ & $2.67^{\mathrm{c}} \pm 2.52$ & $0.90^{\mathrm{c}} \pm 0.80$ \\
\hline & LSD & 4.45 & 1.30 & $\mathbf{0 . 8 0}$ & 0.56 & 0.45 \\
\hline \multirow{4}{*}{ Bahaushe } & 0 & $74.30^{\mathrm{a}} \pm 6.10$ & $40.30^{\mathrm{a}} \pm 5.63$ & $12.80^{\mathrm{a}} \pm 4.50$ & $10.30^{\mathrm{a}} \pm 3.20$ & $4.40^{\mathrm{a}} \pm 0.46$ \\
\hline & 30 & $48.67^{\mathrm{b}} \pm 3.78$ & $28.00^{\mathrm{b}} \pm 5.59$ & $7.47^{\mathrm{b}} \pm 1.27$ & $8.67^{b} \pm 0.58$ & $3.67^{\mathrm{b}} \pm 0.12$ \\
\hline & 60 & $33.67^{\mathrm{c}} \pm 4.64$ & $24.30^{c} \pm 3.79$ & $3.97^{\mathrm{c}} \pm 0.50$ & $7.30^{\mathrm{c}} \pm 0.58$ & $1.87^{\mathrm{c}} \pm 0.15$ \\
\hline & LSD & 4.35 & 2.05 & 1.00 & 0.95 & 0.65 \\
\hline \multirow{4}{*}{ Dandino } & 0 & $79.30^{\mathrm{a}} \pm 13.60$ & $54.20^{\mathrm{a}} \pm 6.42$ & $12.80^{\mathrm{a}} \pm 4.50$ & $20.67^{\mathrm{a}} \pm 4.04$ & $5.70^{\mathrm{a}} \pm 1.60$ \\
\hline & 30 & $58.30^{\mathrm{b}} \pm 7.64$ & $40.90^{\mathrm{b}} \pm 4.40$ & $7.47^{\mathrm{b}} \pm 1.27$ & $8.67^{b} \pm 2.08$ & $4.87^{\mathrm{b}} \pm 0.15$ \\
\hline & 60 & $43.00^{\mathrm{c}} \pm 2.00$ & $33.40^{\mathrm{c}} \pm 2.98$ & $4.00^{c} \pm 0.56$ & $4.67^{\mathrm{c}} \pm 1.53$ & $4.00^{c} \pm 0.10$ \\
\hline & LSD & 5.07 & 2.00 & 1.03 & 0.76 & 0.50 \\
\hline \multirow{4}{*}{ Dan Eka } & 0 & $88.67^{\mathrm{a}} \pm 3.20$ & $61.00^{\mathrm{a}} \pm 5.57$ & $14.67^{\mathrm{a}} \pm 4.07$ & $18.67^{\mathrm{a}} \pm 2.31$ & $5.93^{\mathrm{a}} \pm 0.40$ \\
\hline & 30 & $62.67^{\mathrm{b}} \pm 24.83$ & $48.07^{b} \pm 7.20$ & $10.17^{\mathrm{b}} \pm 2.78$ & $15.30^{\mathrm{b}} \pm 0.58$ & $4.30^{\mathrm{b}} \pm 1.94$ \\
\hline & 60 & $34.67^{c} \pm 13.70$ & $30.30^{c} \pm 8.90$ & $6.60^{\mathrm{c}} \pm 2.90$ & $13.00^{c} \pm 1.00$ & $2.00^{c} \pm 0.30$ \\
\hline & LSD & 4.90 & 1.65 & 1.07 & 1.03 & 0.65 \\
\hline \multirow{4}{*}{ Dan Gombe } & 0 & $58.30^{\mathrm{a}} \pm 6.66$ & $47.67^{b} \pm 0.58$ & $13.60^{\mathrm{a}} \pm 2.14$ & $21.30^{\mathrm{a}} \pm 2.30$ & $3.67^{\mathrm{a}} \pm 0.70$ \\
\hline & 30 & $39.67^{\mathrm{b}} \pm 9.50$ & $33.07^{\mathrm{b}} \pm 2.90$ & $7.80^{\mathrm{b}} \pm 3.90$ & $16.30^{\mathrm{b}} \pm 0.58$ & $2.07^{\mathrm{b}} \pm 0.21$ \\
\hline & 60 & $24.00^{c} \pm 12.53$ & $20.17^{\mathrm{c}} \pm 2.63$ & $3.50^{\mathrm{c}} \pm 0.48$ & $14.30^{\mathrm{c}} \pm 2.08$ & $1.47^{\mathrm{c}} \pm 0.30$ \\
\hline & LSD & 3.68 & 2.03 & 1.00 & 1.04 & 0.39 \\
\hline \multirow[t]{4}{*}{ Dan mazari } & 0 & $33.30^{\mathrm{a}} \pm 4.10$ & $26.67^{\mathrm{a}} \pm 1.53$ & $5.90^{\mathrm{a}} \pm 0.80$ & $16.30^{\mathrm{a}} \pm 0.58$ & $4.20^{\mathrm{a}} \pm 0.21$ \\
\hline & 30 & $28.00^{\mathrm{b}} \pm 1.00$ & $20.50^{\mathrm{b}} \pm 2.10$ & $4.50^{\mathrm{b}} \pm 0.36$ & $14.67^{\mathrm{b}} \pm 0.58$ & $3.10^{\mathrm{b}} \pm 0.10$ \\
\hline & 60 & $23.67^{\mathrm{b}} \pm 0.57$ & $18.00^{\mathrm{b}} \pm 0.30$ & $3.50^{\mathrm{c}} \pm 0.26$ & $12.00^{\mathrm{c}} \pm 1.00$ & $1.87^{\mathrm{c}} \pm 0.50$ \\
\hline & LSD & 4.53 & 2.90 & 0.89 & 0.90 & 0.40 \\
\hline \multirow[t]{4}{*}{ Dan dubukamiya } & 0 & $55.30^{\mathrm{a}} \pm 2.08$ & $48.67^{\mathrm{a}} \pm 1.53$ & $6.77^{\mathrm{a}} \pm 0.21$ & $18.67^{\mathrm{a}} \pm 6.58$ & $5.10^{\mathrm{a}} \pm 0.10$ \\
\hline & 30 & $50.00^{\mathrm{b}} \pm 1.00$ & $44.00^{\mathrm{a}} \pm 1.00$ & $3.80^{\mathrm{b}} \pm 1.32$ & $15.00^{\mathrm{b}} \pm 2.00$ & $4.00^{\mathrm{b}} \pm 0.06$ \\
\hline & 60 & $47.67^{\mathrm{b}} \pm 0.58$ & $38.67^{\mathrm{b}} \pm 2.52$ & $2.60^{\mathrm{b}} \pm 1.40$ & $13.67^{\mathrm{b}} \pm 1.53$ & $3.27^{\mathrm{c}} \pm 0.06$ \\
\hline & LSD & 3.00 & 4.48 & 1.21 & 2.90 & 0.54 \\
\hline \multirow[t]{4}{*}{ Dan kwandawa } & 0 & $23.30^{\mathrm{a}} \pm 1.53$ & $19.67^{\mathrm{a}} \pm 4.73$ & $3.03^{\mathrm{a}} \pm 0.06$ & $7.10^{\mathrm{a}} \pm 1.88$ & $3.67^{\mathrm{a}} \pm 1.53$ \\
\hline & 30 & $19.67^{\mathrm{b}} \pm 4.04$ & $19.67^{b} \pm 1.53$ & $2.40^{\mathrm{a}} \pm 0.04$ & $2.07^{\mathrm{b}} \pm 0.21$ & $2.00^{\mathrm{b}} \pm 1.70$ \\
\hline & 60 & $16.67^{\mathrm{b}} \pm 1.55$ & $10.67^{\mathrm{c}} \pm 0.58$ & $1.67^{\mathrm{a}} \pm 0.10$ & $1.90^{c} \pm 0.93$ & $1.67^{\mathrm{b}} \pm 1.10$ \\
\hline & LSD & 3.04 & 0.90 & 1.50 & 2.01 & 1.00 \\
\hline \multirow[t]{4}{*}{ Ganwon Falke } & 0 & $33.67^{\mathrm{a}} \pm 4.73$ & $20.30^{\mathrm{a}} \pm 2.52$ & $3.70^{\mathrm{a}} \pm 0.67$ & $23.00^{\mathrm{a}} \pm 2.65$ & $4.17^{\mathrm{a}} \pm 0.15$ \\
\hline & 30 & $19.30^{\mathrm{b}} \pm 2.08$ & $17.00^{\mathrm{b}} \pm 1.70$ & $1.37^{\mathrm{b}} \pm 0.12$ & $17.30^{\mathrm{b}} \pm 0.58$ & $3.20^{\mathrm{a}} \pm 0.26$ \\
\hline & 60 & $16.30^{\mathrm{b}} \pm 058$ & $10.30^{\mathrm{c}} \pm 1.50$ & $0.97^{\mathrm{b}} \pm 0.10$ & $14.30^{\mathrm{b}} \pm 0.58$ & $2.00^{\mathrm{a}} \pm 0.10$ \\
\hline & LSD & 5.89 & 2.78 & 1.98 & 3.97 & 2.86 \\
\hline \multirow[t]{4}{*}{ Dan Dogarawa } & 0 & $32.30^{\mathrm{a}} \pm 1.10$ & $23.77^{\mathrm{a}} \pm 3.30$ & $2.77^{\mathrm{a}} \pm 0.99$ & $12.30^{\mathrm{a}} \pm 1.50$ & $3.60^{\mathrm{a}} \pm 0.78$ \\
\hline & 30 & $23.00^{\mathrm{b}} \pm 2.00$ & $18.57^{\mathrm{b}} \pm 1.60$ & $2.27^{\mathrm{a}} \pm 0.76$ & $8.67^{b} \pm 0.58$ & $2.70^{\mathrm{b}} \pm 0.38$ \\
\hline & 60 & $20.00^{\mathrm{c}} \pm 3.60$ & $15.20^{\mathrm{c}} \pm 0.71$ & $0.97^{\mathrm{b}} \pm 1.50$ & $7.00^{\mathrm{b}} \pm 1.00$ & $1.60^{c} \pm 0.30$ \\
\hline & LSD & 2.09 & 1.09 & 0.98 & 1.97 & 0.87 \\
\hline \multirow{4}{*}{ Roma } & 0 & $57.00^{\mathrm{a}} \pm 6.08$ & $46.40^{\mathrm{a}} \pm 5.30$ & $27.10^{\mathrm{a}} \pm 3.80$ & $24.67^{\mathrm{a}} \pm 1.53$ & $5.40^{\mathrm{a}} \pm 0.80$ \\
\hline & 30 & $40.00^{\mathrm{b}} \pm 5.00$ & $40.00^{\mathrm{b}} \pm 5.70$ & $3.87^{\mathrm{b}} \pm 0.98$ & $15.67^{\mathrm{b}} \pm 3.20$ & $4.00^{\mathrm{a}} \pm 0.20$ \\
\hline & 60 & $26.30^{\mathrm{c}} \pm 5.50$ & $20.30^{c} \pm 5.50$ & $1.77^{\mathrm{c}} \pm 0.20$ & $11.67^{\mathrm{c}} \pm 2.51$ & $2.80^{\mathrm{a}} \pm 0.29$ \\
\hline & LSD & 3.87 & 1.09 & 1.98 & 0.43 & 2.08 \\
\hline \multirow[t]{4}{*}{ UTC } & 0 & $69.67 \mathrm{a} \pm 1.53$ & $45.90 \mathrm{a} \pm 5.58$ & $9.00 \mathrm{a} \pm 2.00$ & $13.00 \mathrm{a} \pm 1.70$ & $6.00 \mathrm{a} \pm 0.10$ \\
\hline & 30 & $56.00 \mathrm{a} \pm 14.18$ & $33.00 \mathrm{~b} \pm 3.47$ & $4.67 \mathrm{~b} \pm 1.26$ & $9.00 \mathrm{~b} \pm 1.00$ & $5.10 \mathrm{a} \pm 0.70$ \\
\hline & 60 & $47.30 a \pm 10.02$ & $31.00 \mathrm{~b} \pm 10.02$ & $2.80 \mathrm{c} \pm 1.10$ & $7.30 \mathrm{c} \pm 0.58$ & $3.40 \mathrm{~b} \pm 0.64$ \\
\hline & LSD & 14.87 & 3.42 & 0.54 & 0.76 & 1.53 \\
\hline \multirow{4}{*}{ Rio grande } & 0 & $88.67 \mathrm{a} \pm 3.21$ & $48.10 \mathrm{a} \pm 2.70$ & $5.50 \mathrm{a} \pm 0.90$ & $20.00 \mathrm{a} \pm 3.60$ & $6.70 \pm 0.11$ \\
\hline & 30 & $70.30 \mathrm{~b} \pm 4.50$ & $33.77 \mathrm{~b} \pm 4.00$ & $3.10 \mathrm{~b} \pm 0.30$ & $15.30 \mathrm{~b} \pm 0.58$ & $5.60 \pm 0.46$ \\
\hline & 60 & $51.30 \mathrm{c} \pm 11.00$ & $25.07 \mathrm{c} \pm 2.83$ & $2.50 \mathrm{~b} \pm 0.50$ & $13.00 \mathrm{c} \pm 1.00$ & $4.70 \pm 0.70$ \\
\hline & LSD & 5.12 & 2.07 & 0.67 & 0.75 & 0.87 \\
\hline
\end{tabular}


Table 2 continued. Effects of Salt Concentrations on Phenotypic Parameters in Tomato Genotypes.

\begin{tabular}{|c|c|c|c|c|c|c|}
\hline Genotypes & $\begin{array}{l}\text { Treatment } \\
\left(\mathrm{mgL}^{-1}\right)\end{array}$ & $\begin{array}{lr}\begin{array}{l}\text { Number } \\
\text { leaves } \\
\text { plant) }\end{array} & \begin{array}{r}\text { of } \\
\text { (per }\end{array} \\
\end{array}$ & $\begin{array}{l}\text { Plant } \\
\text { Height } \\
\text { (cm/plant) }\end{array}$ & $\begin{array}{l}\text { Root length } \\
\text { (cm/plant) }\end{array}$ & $\begin{array}{l}\text { Leaf area } \\
(\mathrm{sq} / \mathrm{m})\end{array}$ & $\begin{array}{l}\text { Dry matter } \\
\text { accumulation } \\
\text { (g/plant) } \\
\end{array}$ \\
\hline \multirow[t]{4}{*}{ Giofranco F. } & 0 & $79.00 \mathrm{a} \pm 16.40$ & $47.27 \mathrm{a} \pm 2.64$ & $5.07 \mathrm{a} \pm 0.93$ & $14.67 \mathrm{a} \pm 0.58$ & $5.97 \mathrm{a} \pm 0.29$ \\
\hline & 30 & $56.67 \mathrm{~b} \pm 10.40$ & $33.90 \mathrm{~b} \pm 5.40$ & $3.30 \mathrm{~b} \pm 0.26$ & $12.67 \mathrm{a} \pm 0.58$ & $4.70 \mathrm{~b} \pm 0.70$ \\
\hline & 60 & $33.67 \mathrm{c} \pm 5.51$ & $25.50 \mathrm{c} \pm 4.76$ & $2.80 \mathrm{~b} \pm 0.26$ & $8.67 b \pm 1.10$ & $2.89 c \pm 0.39$ \\
\hline & LSD & 5.70 & 2.97 & 1.31 & 2.53 & 1.02 \\
\hline \multirow[t]{4}{*}{ UC82B } & 0 & $70.30 \mathrm{a} \pm 13.00$ & $44.47 a \pm 5.06$ & $11.40 \mathrm{a} \pm 14.32$ & $16.67 \mathrm{a} \pm 0.57$ & $5.00 \mathrm{a} \pm 0.58$ \\
\hline & 30 & $58.00 \mathrm{~b} \pm 2.60$ & $29.00 \mathrm{~b} \pm 1.70$ & $2.27 b \pm 0.25$ & $14.30 \mathrm{~b} \pm 1.10$ & $4.37 \mathrm{~b} \pm 0.40$ \\
\hline & 60 & $44.30 \mathrm{c} \pm 5.10$ & $23.97 \mathrm{c} \pm 4.38$ & $2.30 \mathrm{~b} \pm 0.60$ & $12.30 \mathrm{~b} \pm 0.57$ & $3.10 \mathrm{c} \pm 0.10$ \\
\hline & LSD & 6.98 & 3.86 & 2.06 & 2.00 & 0.23 \\
\hline \multirow{4}{*}{ Indian tomato } & 0 & $62.30 \mathrm{a} \pm 5.86$ & $41.30 \mathrm{a} \pm 1.50$ & $7.77 a \pm 3.00$ & $9.67 \mathrm{a} \pm 0.57$ & $5.27 \mathrm{a} \pm 0.10$ \\
\hline & 30 & $53.30 \mathrm{~b} \pm 4.90$ & $34.30 \mathrm{~b} \pm 4.00$ & $8.00 \mathrm{a} \pm 0.60$ & $9.00 \mathrm{~b} \pm 0.00$ & $4.17 \mathrm{~b} \pm 0.20$ \\
\hline & 60 & $46.67 \mathrm{~b} \pm 2.89$ & $33.67 \mathrm{~b} \pm 4.00$ & $5.07 \mathrm{~b} \pm 1.08$ & $7.67 \mathrm{c} \pm 50.90$ & $2.00 \mathrm{c} \pm 0.80$ \\
\hline & LSD & 6.87 & 1.98 & 0.94 & 0.56 & 0.50 \\
\hline \multirow[t]{4}{*}{ Tomato peto } & 0 & $59.00 \mathrm{a} \pm 5.29$ & $41.30 \mathrm{a} \pm 1.50$ & $6.00 \mathrm{a} \pm 0.80$ & $21.30 \mathrm{a} \pm 1.50$ & $5.27 \mathrm{a} \pm 0.10$ \\
\hline & 30 & $47.67 \mathrm{~b} \pm 9.40$ & $35.00 \mathrm{~b} \pm 4.00$ & $4.70 \mathrm{~b} \pm 0.38$ & $18.67 \mathrm{~b} \pm 1.10$ & $4.17 \mathrm{~b} \pm 0.20$ \\
\hline & 60 & $43.67 \mathrm{~b} \pm 8.08$ & $29.67 \mathrm{c} \pm 0.58$ & $4.10 \mathrm{c} \pm 1.50$ & $14.30 \mathrm{c} \pm 1.10$ & $2.00 \mathrm{c} \pm 0.80$ \\
\hline & LSD & 0.67 & 1.04 & 0.45 & 0.64 & 0.50 \\
\hline \multirow[t]{4}{*}{ Tropimech } & 0 & $69.30 \mathrm{a} \pm 5.51$ & $38.30 \mathrm{a} \pm 1.00$ & $4.00 \mathrm{a} \pm 0.71$ & $27.30 \mathrm{a} \pm 2.08$ & $6.00 \mathrm{a} \pm 0.56$ \\
\hline & 30 & $64.00 \mathrm{~b} \pm 2.61$ & $33.00 \mathrm{~b} \pm 2.83$ & $3.17 \mathrm{ab} \pm 0.10$ & $21.00 \mathrm{~b} \pm 4.00$ & $5.70 \mathrm{a} \pm 0.61$ \\
\hline & 60 & $53.30 \mathrm{c} \pm 2.52$ & $21.30 \mathrm{c} \pm 1.50$ & $2.80 \mathrm{~b} \pm 0.10$ & $16.00 \mathrm{c} \pm 1.70$ & $4.40 \mathrm{~b} \pm 0.66$ \\
\hline & $\begin{array}{l}60 \\
\text { LSD }\end{array}$ & 3.23 & 2.43 & 0.98 & 1.00 & 0.76 \\
\hline \multirow[t]{4}{*}{ Cherry } & 0 & $33.67 \mathrm{a} \pm 3.20$ & $28.87 a \pm 3.59$ & $7.87 \mathrm{a} \pm 2.20$ & $15.30 \mathrm{a} \pm 0.58$ & $4.17^{\mathrm{a}} \pm 0.12$ \\
\hline & 30 & $24.30 \mathrm{~b} \pm 3.79$ & $25.17 \mathrm{~b} \pm 0.90$ & $4.07 \mathrm{~b} \pm 2.50$ & $13.30 \mathrm{~b} \pm 1.50$ & $3.10^{\mathrm{b}} \pm 0.10$ \\
\hline & $\begin{array}{l}50 \\
60\end{array}$ & $21.00 \mathrm{c} \pm 2.00$ & $19.43 c \pm 3.60$ & $1.97 \mathrm{c} \pm 0.35$ & $12.67 \mathrm{~b} \pm 1.50$ & $2.00^{\mathrm{c}} \pm 0.10$ \\
\hline & $\begin{array}{l}60 \\
\text { LSD }\end{array}$ & 1.67 & 1.54 & 0.34 & 0.94 & 0.45 \\
\hline \multirow{4}{*}{ Heirloom } & 0 & $66.67 \mathrm{a} \pm 3.50$ & $43.23 \mathrm{a} \pm 2.90$ & $5.70 \mathrm{a} \pm 0.80$ & $16.67 \mathrm{a} \pm 2.08$ & $5.30 \mathrm{a} \pm 0.30$ \\
\hline & 30 & $55.00 \mathrm{~b} \pm 2.60$ & $37.87 \mathrm{~b} \pm 1.30$ & $4.17 \mathrm{~b} \pm 0.50$ & $9.00 \mathrm{~b} \pm 1.70$ & $4.30 \mathrm{~b} \pm 0.43$ \\
\hline & 60 & $46.00 \mathrm{c} \pm 4.00$ & $31.07 \mathrm{c} \pm 1.96$ & $3.20 \mathrm{c} \pm 0.20$ & $7.00 \mathrm{c} \pm 0.00$ & $3.00 \mathrm{c} \pm 0.10$ \\
\hline & LSD & 4.45 & 2.76 & 0.74 & 0.89 & 0.54 \\
\hline
\end{tabular}

Values represent means and standard deviation of vegetative parameters. Mean in a column with the same superscript are not significantly different at $(\mathrm{P}<0.05)$.

\section{Allelic Variation among the Polymorphic Simple Sequence Repeat Loci}

A total of 144 alleles were detected including 2 rare alleles with no unique allele. The cgSSR from XM_010323394.1 gene produced the lowest number of 4 alleles, followed by NM_001287774.1, AI486387.1, AY562123.1 with 5 alleles. The cgSSR from AI773078.1 gene gave rise to the highest number of alleles (19). In this research, only di-tetra nucleotide repeats and reiteration of motifs less than 5 times was excluded. Di -nucleotide motifs were found to be the largest with 175 SSR loci and tetra-nucleotide motifs formed the smallest group with 48 SSR loci. List of those genes with SSR loci with their respective gene bank LOC number, function, number, types and location of motif found were detailed in Table 3. 
Table 3. Details of Salt Tolerant Gene LOC Number, Motifs with Repeats Number, Location in Sequence, Primers with Molecular Weight.

\begin{tabular}{|c|c|c|c|c|c|c|c|c|}
\hline $\begin{array}{l}\text { Gene Bank } \\
\text { LOC } \\
\text { Number } \\
\end{array}$ & Forward & Reverse & $\begin{array}{l}\text { Location } \\
\text { of Motif }\end{array}$ & Function & $\begin{array}{l}\text { Expected } \\
\text { amplicon } \\
\text { size } \\
\end{array}$ & Motif & $\begin{array}{l}\text { PIC } \\
\text { Value } \\
\end{array}$ & $\begin{array}{l}\text { No. of } \\
\text { Alle- } \\
\text { lle }\end{array}$ \\
\hline $\begin{array}{l}\text { XM_0103233 } \\
94.1\end{array}$ & $\begin{array}{l}\text { GACCATTATGTTGTTTGGTG } \\
\text { CCG }\end{array}$ & $\begin{array}{l}\text { AGAGGTCCAACTTCTGGATC } \\
\text { GCAT }\end{array}$ & CDS & antiporter & 169 & $(\text { at })_{3}$ & 0.04 & 2 \\
\hline $\begin{array}{l}\text { NM_0012877 } \\
74.1\end{array}$ & $\begin{array}{l}\text { GCTGGGATGAGTGGAGCTG } \\
\text { A }\end{array}$ & $\begin{array}{l}\text { TCCAAGTGAGCCCTTTTTGG } \\
\text { GAT }\end{array}$ & CDS & $\begin{array}{l}\text { Water } \\
\text { Transport }\end{array}$ & 376 & $(\mathrm{cct})_{3}$ & 0.063 & 2 \\
\hline KM094129.1 & $\begin{array}{l}\text { GCCAAATTACGCGTGTGATT } \\
\text { CTGT }\end{array}$ & $\begin{array}{l}\text { CAGTTCGGATGACCTTGCAT } \\
\text { TCATA }\end{array}$ & CDS & $\begin{array}{l}\text { Transcription } \\
\text { Factor }\end{array}$ & 151 & $(\operatorname{atg})_{3}$ & 0.303 & 2 \\
\hline $\begin{array}{l}\text { NM_0012789 } \\
76.2\end{array}$ & $\begin{array}{l}\text { GCAACTGCTGTCTTCAGCAC } \\
\text { TGTAT }\end{array}$ & $\begin{array}{l}\text { GAACTCTGCAAAATCACTTC } \\
\text { ACCCT }\end{array}$ & CDS & Signaling & 241 & $(\text { gaa })_{4}$ & 0.123 & 3 \\
\hline AI773078.1 & $\begin{array}{l}\text { GAT GGA CAC CCT TCA ATT } \\
\text { TAT GGT }\end{array}$ & $\begin{array}{l}\text { TCC AAG TAT CAG GCA CAC } \\
\text { CAG C }\end{array}$ & Intronic & $\begin{array}{l}\text { RNA } \\
\text { processing }\end{array}$ & 145 & (aat) 14 & 0.903 & 4 \\
\hline AI778183.1 & $\begin{array}{l}\text { GCG AAG AAG ATG AGT } \\
\text { CTA GAG CAT AG }\end{array}$ & $\begin{array}{l}\text { CTC TCT CCC ATG AGT TCT } \\
\text { CCT CTT C }\end{array}$ & 3'UTR & $\begin{array}{l}\text { RNA } \\
\text { processing }\end{array}$ & 120 & (aat) 12 & 0.123 & 2 \\
\hline AW037347.1 & $\begin{array}{l}\text { GCC ACG TAG TCA TGA TAT } \\
\text { ACA TAG }\end{array}$ & GCC TCG GAC AAT GAA TTG & 5'UTR & $\begin{array}{l}\text { RNA } \\
\text { processing }\end{array}$ & 180 & (aat) 12 & 0.203 & 2 \\
\hline AI491065.1 & $\begin{array}{l}\text { ACT GCA TTT CAG GTA CAT } \\
\text { ACT CTC }\end{array}$ & $\begin{array}{l}\text { ATA AAC TCG TAG ACC ATA } \\
\text { CCC TC }\end{array}$ & CDS & $\begin{array}{l}\text { Regulatory, } \\
\mathrm{Ca} 2+\text { binding }\end{array}$ & 200 & (at)9 & 0.49 & 4 \\
\hline AW034362.1 & $\begin{array}{l}\text { CCG CCT CTT TCA CTT GAA } \\
\text { C }\end{array}$ & $\begin{array}{l}\text { CCA GCG ATA CGA TTA GAT } \\
\text { ACC }\end{array}$ & CDS & $\begin{array}{l}\text { Transcription } \\
\text { Factor }\end{array}$ & 130 & $(\mathrm{cag}) 7$ & 0.203 & 3 \\
\hline AI780156.1 & $\begin{array}{l}\text { TCC AAT TTC AGT AAG } \\
\text { GAC CCC TC }\end{array}$ & $\begin{array}{l}\text { CCG AAA ACC TTT GCT ACA } \\
\text { GAG TAG A }\end{array}$ & 3'UTR & Signaling & 150 & (ct) 12 & 0.16 & 3 \\
\hline AI895126.1 & $\begin{array}{l}\text { GCT CTG TCC TTA CAA ATG } \\
\text { ATA CCT CC }\end{array}$ & $\begin{array}{l}\text { CAA TGC TGG GAC AGA AGA } \\
\text { TTT AAT G }\end{array}$ & Intronic & $\begin{array}{l}\text { regulatory, } \\
\text { Helicase }\end{array}$ & 160 & $(\mathrm{ta}) 9$ & 0.423 & 4 \\
\hline AW031453.1 & $\begin{array}{l}\text { GCC GTT CTT GGT GGA TTA } \\
\text { G }\end{array}$ & $\begin{array}{l}\text { CCT CCT TTC GTG TCT TTG } \\
\text { TC }\end{array}$ & 5'UTR & $\begin{array}{l}\text { regulatory, } \\
\text { Helicase }\end{array}$ & 300 & (ta) 20 & 0.563 & 3 \\
\hline $\begin{array}{l}\text { AQ368062.1 } \\
\text { AI486387.1 }\end{array}$ & $\begin{array}{l}\text { TGA TCC TAA GCT TTT TCC } \\
\text { GTG AGT } \\
\text { ACG CTT GGC TGC CTC GGA }\end{array}$ & $\begin{array}{l}\text { CAA GTT CAC CTC ATT TCA } \\
\text { CCC CT } \\
\text { AAC TTT ATT ATT GCC ACG } \\
\text { TAG TCA TGA }\end{array}$ & $\begin{array}{l}\text { CDS } \\
\text { 3'UTR }\end{array}$ & $\begin{array}{l}\text { regulatory, } \\
\text { Helicase } \\
\text { Balances the } \\
\text { concentration } \\
\text { of myo- } \\
\text { inositol }\end{array}$ & $\begin{array}{l}350 \\
250\end{array}$ & $\begin{array}{l}\text { (ta) } 19 \\
\text { tat) } 12\end{array}$ & $\begin{array}{l}0.64 \\
0.01\end{array}$ & $\begin{array}{l}5 \\
2\end{array}$ \\
\hline AY562123.1 & $\begin{array}{l}\text { CCT GTT GAT GCC AAT AAT } \\
\text { CAA A }\end{array}$ & $\begin{array}{l}\text { ATT CCA CTC AAC CCA ACA } \\
\text { AAT G }\end{array}$ & 5'UTR & $\begin{array}{l}\text { Fuctional } \\
\text { Anti } \\
\text { oxidation }\end{array}$ & 200 & (ta) 10 & 0.063 & 2 \\
\hline
\end{tabular}

\section{Genetic Diversity Analysis Based on SSR}

The data matrix generated from $15 \mathrm{cgSSRs}$ profiling of 20 genotypes were utilized to study the genetic diversity by cluster analysis. The dendrogram generated through unweighted pair group method of arithmetic mean (UPGMA) showed the similarity among the tomato genotype. The dendrogram exhibited four distinct clusters, interestingly none of the genetypes from different panel (i.e accessions and landraces) intermixed with one another (Fig. 1). It was observed that dandino, dan kwandawa, dan mazari, dan eka and dan dubukamiya having same collection locality formed a separate subgroup. However, the same pattern of distinct subgroup was obtained under a subgroup with UTC and UC82B accessions. It is distinct from the genetic diversity analysis using the 15 cgSSR markers that those markers are able to distinguish tomato genotypes on the basis their genetic diversity based on salinity responsive genes (Fig. 1). 


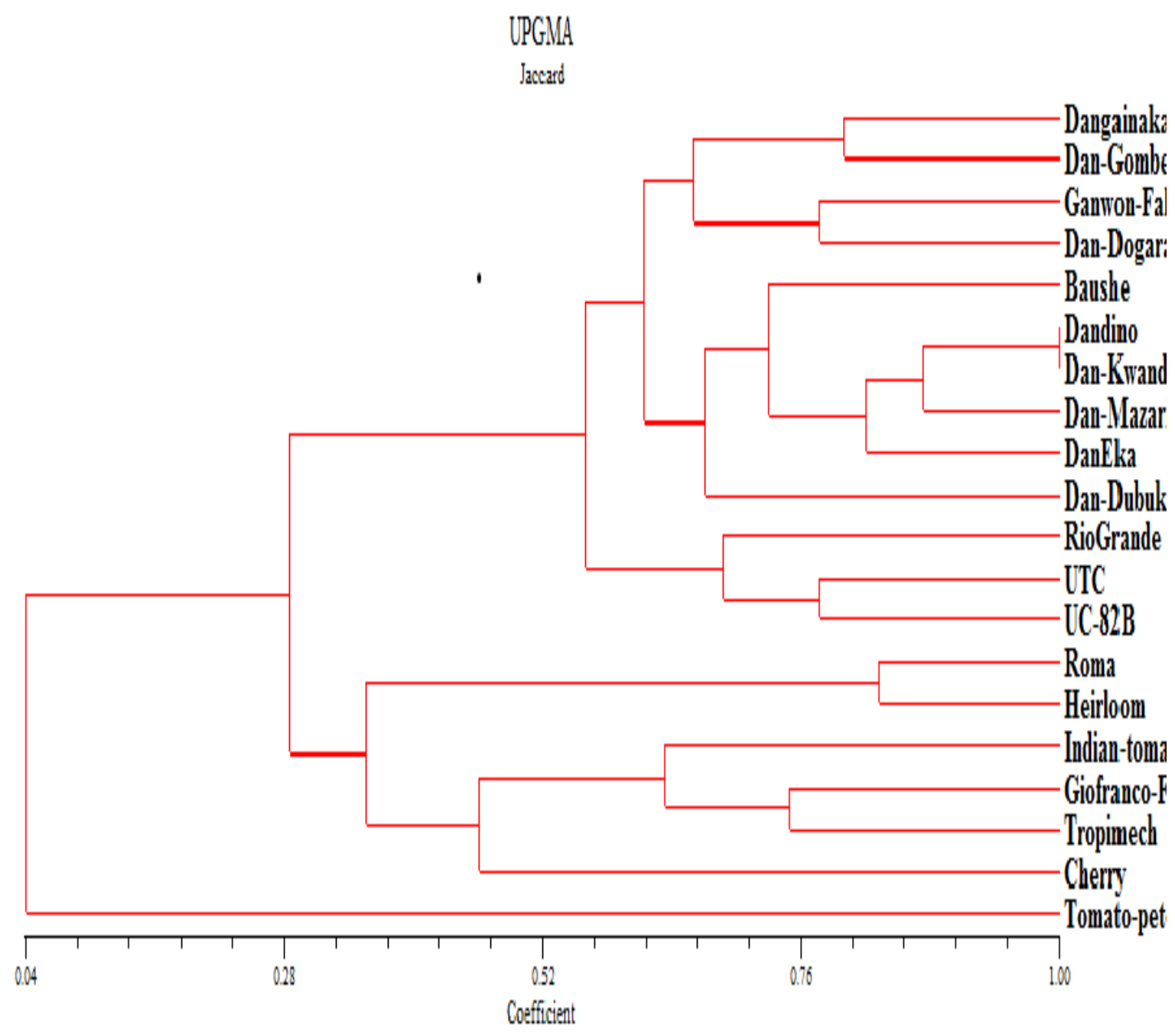

Figure 1. Dendrogram Based on Salinity Responsive Gene in Tomato Genotype.

\section{Discussions}

In this study it was observed that salinity decreased vegetative parameters with increase in salt concentration. In all the twenty genotypes studied, control had the highest vegetative parameters (number of leaves, plant height, leaf area, root length and dry matter accumulation) followed by plants treated with $30 \mathrm{mgL}^{-1}$ of salt. The lowest vegetative parameters were observed on plants treated with $60 \mathrm{mgL}^{-1}$ of salt. Control had the highest number of leaves, plant height, root length, leaves area and dry matter accumulation. This is in accordance with the findings of [17] on effect of growth biochemical parameters and ion homeostasis in Solanum lycopersicum. Root and shoot lengths are the most important parameters for salt stress because of the direct contact of root with the soil and absorb water and shoot supply to the rest of plant body. For this reason, root and shoot length provides an important clue to the response of plant to salt stress (Muhammad et al., 2006). As salinity is first perceived in the root, it is likely that root derived signal, presumably abscisic acid is formed which directly or indirectly down regulates leaf expansion rate [19-20].

\section{Phylogenetic Analysis of Tomato Using Simple Sequence Repeats Markers}

In the phylogenetic analysis, most of the tomato landraces and accessions were clustered together in respect to their genetic variation in response to salinity responsive gene, and might have a similar genetic background. Those clustered within the same group or subgroups are mostly from the same origin and those, which are distantly grouped, might be genetically distinct. The relationship was also observed in similarity of the landraces genotypes in terms of their growth 
habit. In group IV, Bahaushe, Dandino and Dan eka are from the same location and exhibited same growth habit. Similar result was reported by [16] on tomato. Cultivars from same geographical locations were group in a cluster of the dendrogram.

\section{Conclusion}

In conclusion, SSR based dendrogram showed clear relationship among the two panels (i.e accessions and landraces) in group I. interestingly, none of the landraces intermixed with the accessions in a sub group. This could help to improve genetic diversity analysis in tomato and the markers obtained could be used in a wide range of identification and pre-screening for salinity responsive gene in tomato.

\section{Conflict of Interest}

The authors declare that there is no conflict of interest.

\section{Acknowledgement}

Sincere appreciation and gratitude to Dr. Kutub Ali-Molla for his advice and support toward the completion of this research. Our gratitude also goes to Dr. Abubakar Muhammad Gumi for his support in analysis of the result.

\section{References}

[1] J. Singh, E.V.D. Sastry, V. Singh, Effect of salinity on tomato (Lycopersicon esculentum Mill.) during seed germination stage, Journal of Plant Physiology and Molecular Biology. 18 (2012) $45-50$.

[2] X. Zhang et al., Recent progress in drought and salt tolerance studies in Brassica crops, Journal of Science Breeding. 64 (2014) 60-73.

[3] H.J. Bohnert, D.E. Nelson, R.G. Jensen, Adaption of Environmental Stresses, Plant and Cell. 7 (1995) 1099-1111.

[4] C. James (2006). Global Status of Transgenic Crops, Various Global Review Briefs from 1996 to 2006. International Service for the Acquisition of Agri-Biotech Application (ISAAA).

[5] R. Munns, Comparative physiology of salt and water stress, Journal of Plant Cell Environment. 25 (2002) 239-250.

[6] S. Benor et al., Assessment of genetic variation in tomato (Solanum lycopersicum L.) inbred lines using SSR molecular markers, Journal of Genetics and Genomics. 35 (2008). 373-379.

[7] G. Levinson, G.A. Gutman, Slipped-strand mispairing: A major mechanism for DNA sequence evolution, Journal of Molecular Biology and Evolution. 4 (1987) 203-221

[8] H. Hamada et al., Characterization of genomic poly $(\mathrm{dT}-\mathrm{dG})$ poly $(\mathrm{dC}-\mathrm{dA})$ sequences: Structure, organization and conformation, Journal of Molecular and Cell Biology. 4 (1984) 2610-2621.

[9] J. Van der Schoot, M. Pospiskova, B. Vosman, Development and characterization of microsatellite markers in black poplar (Populus nigra L.), Theoretical and Applied Genetics. 101 (2000) 317-322.

[10] H. Zhou, T. Hanson, J. Zhang, Generalized accelerated failure time spatial frailty model for arbitrarily censored data, Lifetime Data Analogue. 23(3) (2016) 495-515.

[11] E. Asare-Bediako et al., Tomato Production Constraints at Bontanga Irrigation Project in the Northern Region of Ghana, Journal of Applied Sciences. 7 (2007) 459-461. 
[12] FOA, International conference on agricultural statistics VII. Mordernization of Agricultural Statistics in Support the sustainable Development Agenda. Adis Ababa, 2013, pp. 45-46.

[13] L.E. Allison, Preliminary Report. Salinity Status of Irrigation Schemes of Northern Nigeria. USAID, Consultants Report N. 14, 1962.

[14] IPGRI, Laboratory Bench activity glossary. How to calculate Leaf Surface area, 2014, pp. 2729.

[15] K. Ali-Molla et al., Identification and analysis of novel salt responsive candidate gene based SSRs (cgSSRs) from rice Oryza sativa L.), BMC Plant Journal of Biology. 15 (2015) 122130.

[16] D. Botstein et al., Construction of a Genetic Linkage Ma using restriction Fragment length Polymorphisms, American Journal of Human Gene. 32(3) (1980) 314-331.

[17] A.M. Gumi et al., Salinity Stress: Effects on growth, biochemical parameters and ion homeostasis in Solanum lycopersicum L. (Cv. Daneka), Central European Journal of Experimental Biology. 2(3) (2013) 20-25.

[18] J. Muhammad et al., Studies on germination and growth of cabbage, sugar beet, paniculate and pak-choi, Journal of Central European Agriculture. 7 (2006) 273-281.

[19] J.B. Passioura, Root signals control leaf expansion in wheat seedlings growing in drying soil, Australian Journal of Plant Physiology. 15 (1988) 687-693.

[20] H. Zhao et al., Rheological and Sensory Properties of tomato Varieties from Improvement Programmes in West Africa, International Food Journal. 16 (1991) 167- 174.

[21] C. He, V. Poysa, K. Yu, Development and Characterization of Simple Sequence Repeat (SSR) Markers and their Use in Determining Relationships among Lycopersicon esculentum cultivars, Theoretical and Applied Genetics. 106 (2003) 363-373. 